\title{
Superquadrics Parameter Estimation from Shading Image Using Genetic Algorithm
}

\author{
Hideo SAITO \\ saikin@ozawa.elec.keio.ac.jp \\ tuna@ozawa.elec.keio.ac.jp \\ Department of Electrical Engineering, Keio University \\ 3 - 14 - 1 Hiyoshi Kouhoku-ku Yokohama 223, Japan \\ phone: +81 - 45 - 563 - 1141 (ext.3309), fax: + 81 - 45 - 563 - 2773
}

Nobuhiro TSUNASHIMA

\begin{abstract}
D shape modeling is very important for efficient shape description and recognition. Superquadrics that is a parametric 3-D shape modeling function can represent various shapes by using a single equation with some parameters. In this study, the superquadrics parameters of 3-D shape are estimated from a 2-D shading image by using genetic algorithm (GA), which is an optimizing technique based on mechanisms of natural selection. Ten parameters, which are five parameters of the superquadrics shape, three eular angle parameters, and two shift parameters, are coded as a string in the GA. The string is evaluated by the difference between the given 2-D shading image and the calculated shading image from the 3-D shape represented by the parameters. By applying the GA to the optimization of the evaluation value, the string having the minimum difference is sought. The parameters are estimated from some shading images of various 3-D shapes by using the proposed method, and the results are presented.
\end{abstract}

\section{INTRODUCTION}

The shape from shading [1], which is the method for reconstructing 3-D shape of an object from 2-D shading image has long been studied in the field of computer vision. Since a $2-\mathrm{D}$ shading image cannot determine the 3-D shape uniquely, many researchers have tried to reconstruct the $3-\mathrm{D}$ shapes by using some constraints on the object shapes, such as the boundary conditions of the object shape, smooth constraint of the surface, etc.

In our previous study [2], we regarded the problem of the shape from shading as a kind of optimization problem, which is to search for the shape providing the most similar shading image to the shading image of the original object, and then genetic algorithm (GA) was applied to the optimization problem. In this method, however, the reconstructed shapes have some random noises because there are too many shape candidates to determine the shape of the object uniquely.

In this paper, we reduce the shape from shading problem to 3-D parametric model from shading. The $3-\mathrm{D}$ shape modeling [3] is very important issue in the study of computer vision and computer graphics. We employ the superquadrics for 3-D parametric model [4][5][6], which are proposed for representing 3-D smooth surfaces by using primitives expressed as a simple parametric equation. A complicated shape can be described by several parameters of the primitives that are represented by superquadrics. By using the superquadrics model as the parametric model, shape reconstruction can be reduced to parameter estimation. Then the parameters can be uniquely determined from a 2-D shading image.

As mentioned above, the objective of the study is to get superquadrics model of the $3-\mathrm{D}$ shape from a $2-\mathrm{D}$ shading image. The estimated parameters are ten; five is the parameter in the superquadrics equation, three parameters represent the orientation of the object shape, and two represent the location of the object. The estimation of the ten parameters at the same time is difficult by using the conventional optimizing method like hill climbing approach and so on. In this study, therefore, genetic algorithm (GA) [7][8] which is an optimizing techniques based on mechanisms of natural selection, is applied for estimating the parameters. The advantages of GA are: (1) The solution is not likely to be forced into the local optimal points, since the optimal search of GA is principally a kind of multi-point searching. (2) An optimized evaluation function is flexibly designed because the evaluation functions are not required to be differentiable and analyzable.

In this paper, the principle of the proposed method for estimating the parameters of a 3 -D shape from a 2-D shading image using GA is described. For demonstration of the proposed method, model parameters of 3-D shapes are estimated from shading images that are obtained by computer simulation.

\section{SHADING IMAGES}

On the shading images in this study, we supposed the following conditions:

1. The object surface is Lambertian.

2. The direction of the light source and the view point are same.

3. The imaging system is orthographic projection.

Under the conditions, the gray levels in a shading image $S(x, y)$ are determined by only the orientation of the object surface, which is expressed as the following equation.

$$
S(x, y)=r_{0} \cos i
$$

where $r_{0}$ represents surface reflectance constant, and $i$ is the angle between the normal vector of the surface and the direction of the light source ( $Z$ direction) as shown in figure 1 . 


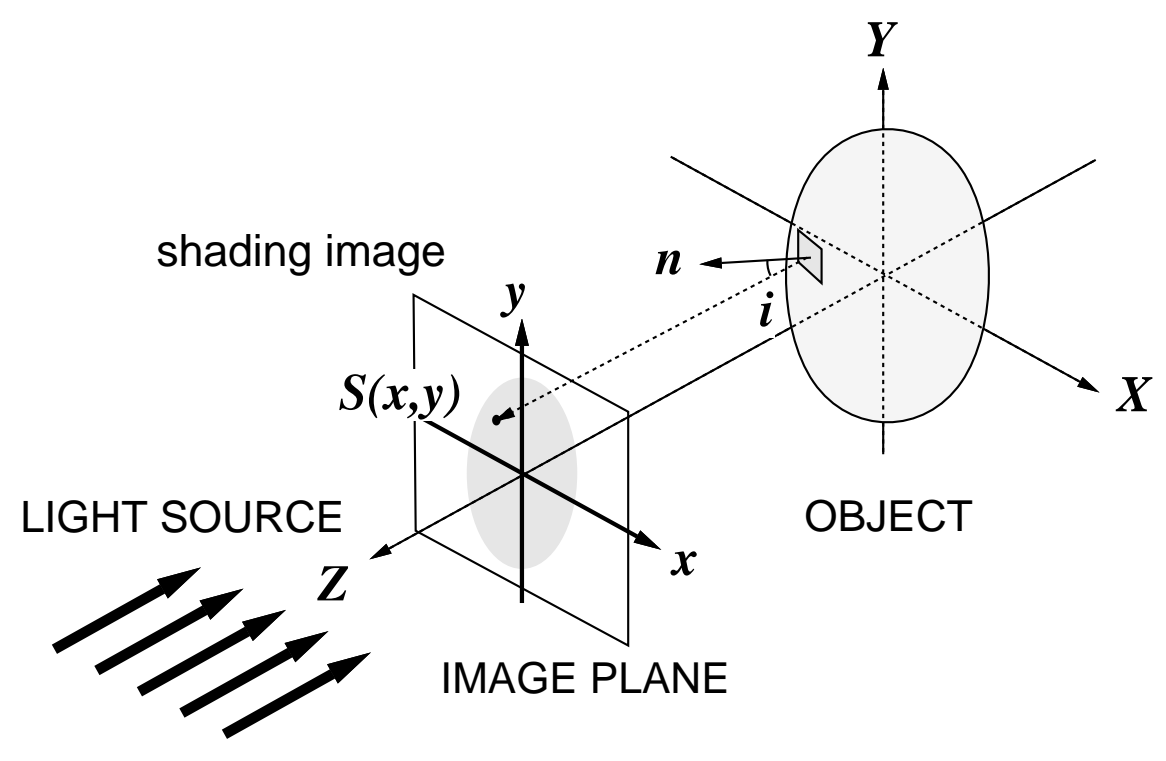

Figure 1: The definition of the coordinate system of the object and the shading image. The shading image is obtained under the orthographic projection. The direction of the light source is parallel to the $Z$ axis.
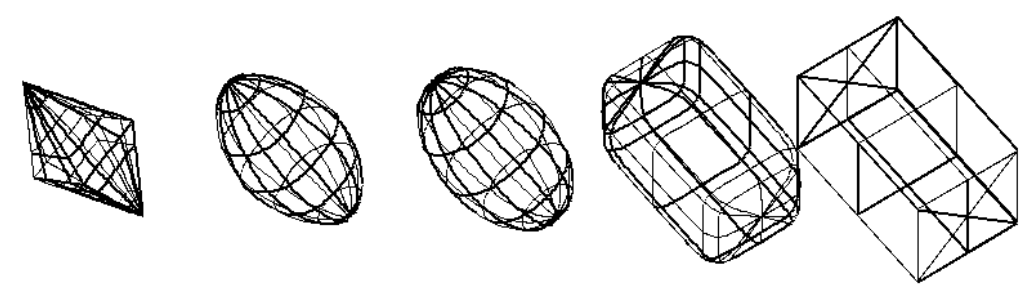

$\varepsilon=2.0$

$\varepsilon=1.0$

$\varepsilon=0.01$

Figure 2: The various shapes of superquadrics for various $\varepsilon$.

\section{SUPERQUADRICS}

The superquadrics is an extension of quadratic surface. The complicated shapes can be described by several parameters of the primitives which are represented by superquadrics. In this way, the superquadrics can be applicable to not only computer graphics but also computer vision.

The equation of the superquadrics is expressed as

$$
f(x, y, z)=\left\{\left(\frac{x}{a_{1}}\right)^{\frac{2}{\varepsilon_{2}}}+\left(\frac{y}{a_{2}}\right)^{\frac{2}{\varepsilon_{2}}}\right\}^{\frac{\varepsilon_{2}}{\varepsilon_{1}}}+\left(\frac{z}{a_{3}}\right)^{\frac{2}{\varepsilon_{1}}}=1,
$$

where $a_{1}, a_{2}$, and $a_{3}$ are scaling parameters in $x, y$, and $z$ directions, respectively, and $\varepsilon_{1}$ and $\varepsilon_{2}$ are squareness parameters. In figure 2 , The various shapes of superquadrics for various $\varepsilon$ are shown.

\section{Rotation and Shift of 3-D Object}

In equation (2), superquadrics surface is defined in an object centered coordinate system $\left(X_{c}, Y_{c}, Z_{c}\right)$. An actual object surface must be expressed in a world coordinate system $(X, Y, Z)$. The relationship between $(X, Y, Z)$ and $\left(X_{c}, Y_{c}, Z_{c}\right)$ is defined with a homogeneous coordinate transform $\boldsymbol{T}$ as the following:

$$
\left[\begin{array}{c}
X \\
Y \\
Z \\
1
\end{array}\right]=\boldsymbol{T}\left[\begin{array}{c}
X_{c} \\
Y_{c} \\
Z_{c} \\
1
\end{array}\right]=\boldsymbol{S R}_{x} \boldsymbol{R}_{y} \boldsymbol{R}_{z}\left[\begin{array}{c}
X_{c} \\
Y_{c} \\
Z_{c} \\
1
\end{array}\right]
$$

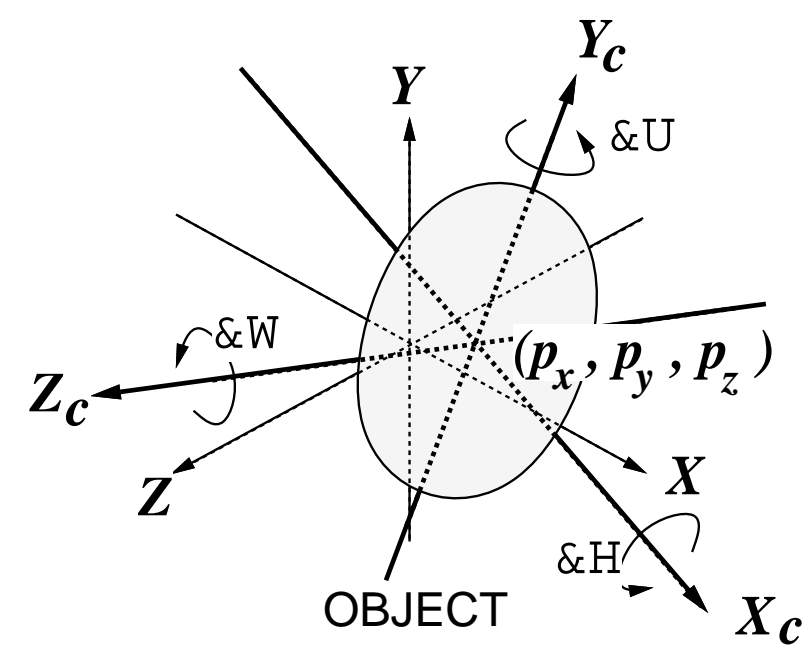

Figure 3: The definition of Euler angle parameters $(\theta, \phi, \psi)$ and

\begin{tabular}{|c|c|c|c|c|c|c|c|c|}
\hline$P Y|P X|$ & $D Z$ & $D Y$ & $D X$ & $A_{3}$ & $A_{2}$ & $A_{I}$ & $\boldsymbol{E}_{2}$ & $\boldsymbol{E}_{1}$ \\
\hline \multicolumn{9}{|c|}{ 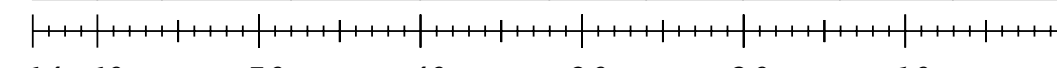 } \\
\hline 6460 & 5 & & 40 & 30 & & 0 & 10 & \\
\hline
\end{tabular}
shift parameters $\left(p_{x}, p_{y}, p_{z}\right)$.

Figure 4: Scheme for coding.

where $S$ represents the shift of the center of the coordinate $\left(X_{c}, Y_{c}, Z_{c}\right) ; \boldsymbol{R}_{x}, \boldsymbol{R}_{y}$, and $\boldsymbol{R}_{z}$ represent the rotation around the axis of $X_{c}, Y_{c}$, and $Z_{c}$ by angle $\theta, \phi$, and $\psi$, respectively. These matrices are expressed as

$$
\begin{gathered}
\boldsymbol{S}=\left[\begin{array}{llll}
1 & 0 & 0 & p_{x} \\
0 & 1 & 0 & p_{y} \\
0 & 0 & 1 & p_{z} \\
0 & 0 & 0 & 1
\end{array}\right], \\
\boldsymbol{R}_{x}=\left[\begin{array}{cccc}
1 & 0 & 0 & 0 \\
0 & \cos \theta & -\sin \theta & 0 \\
0 & \sin \theta & \cos \theta & 0 \\
0 & 0 & 0 & 1
\end{array}\right], \\
\boldsymbol{R}_{y}=\left[\begin{array}{cccc}
\cos \phi & 0 & \sin \phi & 0 \\
0 & 1 & 0 & 0 \\
-\sin \phi & 0 & \cos \phi & 0 \\
0 & 0 & 0 & 1
\end{array}\right] \\
\boldsymbol{R}_{z}=\left[\begin{array}{cccc}
\cos \psi & -\sin \psi & 0 & 0 \\
\sin \psi & \cos \psi & 0 & 0 \\
0 & 0 & 1 & 0 \\
0 & 0 & 0 & 1
\end{array}\right]
\end{gathered}
$$

This equation represents that a point is first rotated by eular angle $(\theta, \phi, \psi)$ and then shifted by $\left(p_{x}, p_{y}, p_{z}\right)$ using the transformation $\boldsymbol{T}$. Accordingly, superquadrics for general orientation and position can be expressed by Euler angle parameters $(\theta, \phi, \psi)$ and shift parameters $\left(p_{x}, p_{y}, p_{z}\right)$, in addition to the shape parameters $\left(a_{1}, a_{2}, a_{3}, \varepsilon_{1}, \varepsilon_{2}\right)$ shown in equation (2). Definition of these parameters are shown in figure 3 . 


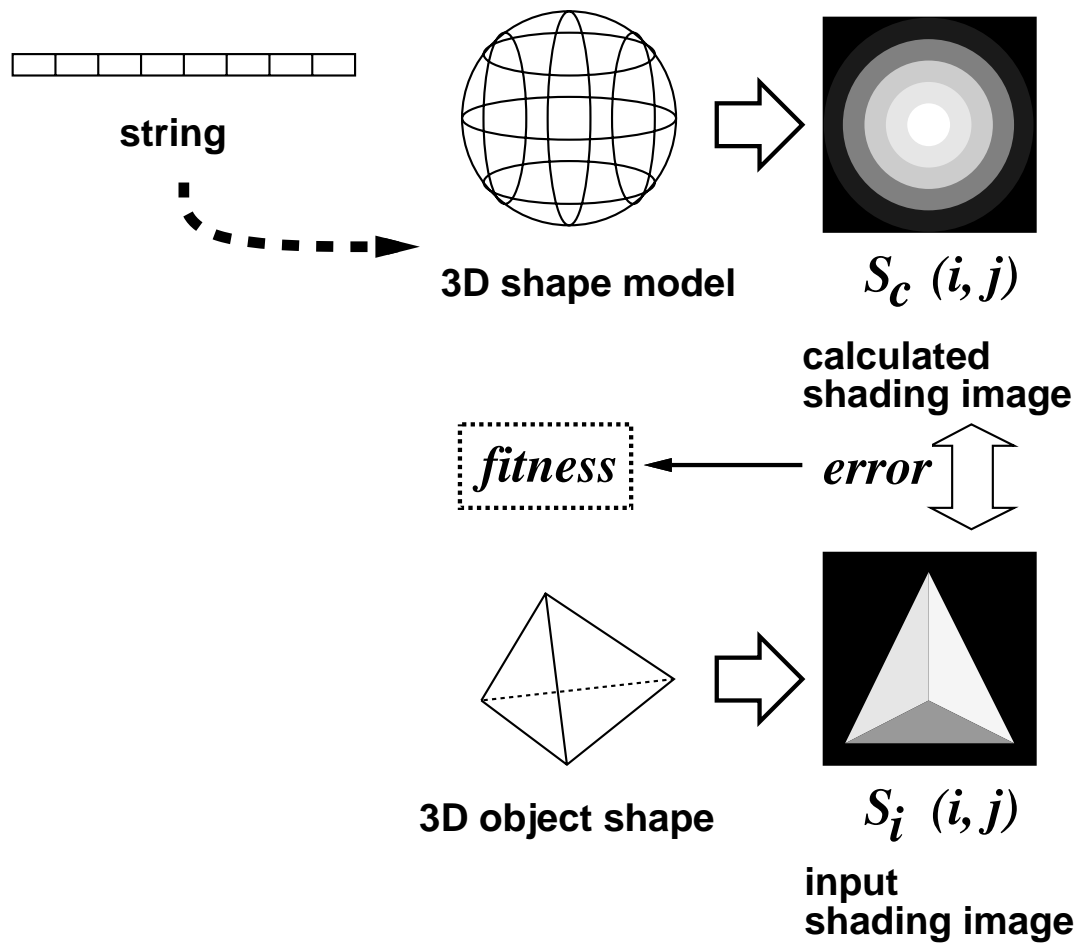

Figure 5: The fitness value is defined according to the similarity between the shading image determined by the 3 -D shape and the given (input) shading image.

\section{Parameter Estimation From Shading IMAGE USING GA}

In this study, the parameters representing superquadrics for general orientation and position are estimated from a 2-D shading image. The shading image is independent of $p_{z}$, because the orthographic projection imaging system is used as shown in figure 1. Therefore $p_{z}$ is excluded for estimation. Then ten parameters which are shown below are used for representing the superquadrics model of the 3-D object shape, and estimated by using the optimizing function of GA.

1. Model parameters of superquadrics $\left(\varepsilon_{1}, \varepsilon_{2}, a_{1}, a_{2}, a_{3}\right)$

2. Eular angle of 3-D object $(\theta, \phi, \psi)$

3. Shift of $3-\mathrm{D}$ object $\left(p_{x}, p_{y}\right)$

\section{Coding and Decoding of the Parameters}

For applying GA, the candidates of solution must to be coded and then represented by strings. In this method, the ten parameters to be optimized are coded as binary representation, and they are cascaded each other. Figure 4 shows the scheme for coding.

The binary representations are decoded according to the following equations.

$$
\varepsilon_{n}=2 \frac{6.0\left(128.0-E_{n}\right)}{128.0}-5.0 \quad(n=\{1,2\})
$$

where $E_{n}$ is 7 bits integer $\left(0 \leq E_{n} \leq 128\right)$, and then $2^{-5} \leq \varepsilon_{n} \leq 2$.

Since the orthographic projection is supposed as image formation system, only the relative size of the object can be estimated. Hence, the scale of the object in $X$ and $Y$ direction is defined as the same as the image plane $(x-y$ plane). When the size of the
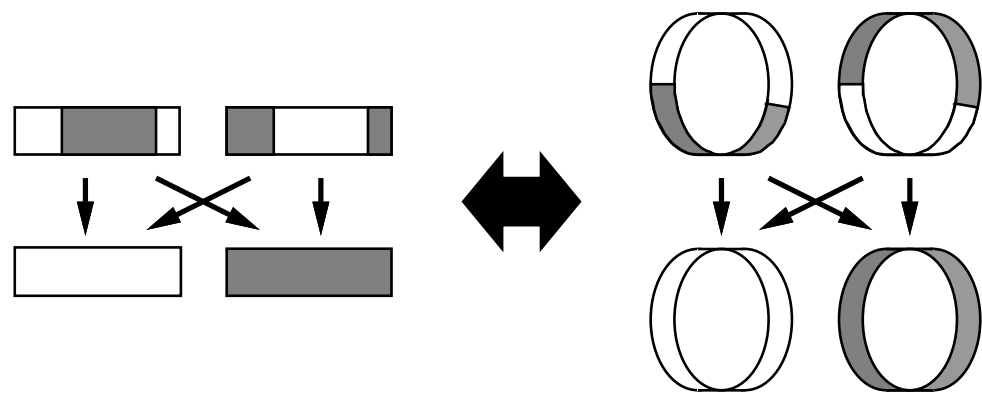

Figure 6: Scheme of the two point crossover.

image plane is considered as $32 \times 32$ arbitrary unit, the scale parameters can be considered as 16 in maximum. Accordingly, scale parameters $a_{1}, a_{2}, a_{2}$ are

$$
a_{n}=10.0\left(\frac{A_{n}}{64.0}+0.5\right) \quad(n=\{1,2,3\})
$$

where $A_{n}$ is 6 bits integer $\left(0 \leq A_{n} \leq 63\right)$, and then $5 \leq a_{n} \leq 15$.

For the orientation parameters,

$$
\theta=\frac{D X}{128.0}
$$

where $D X$ is 8 bits integer $(0 \leq D X \leq 255)$, and then $0 \leq \theta \leq 2 \pi$. Decoding of $\phi$ and $\psi$ are the same.

For the shift parameters,

$$
p_{x}=\frac{(P X-8.0)}{4.0}+G X
$$

where $P X$ is 4 bits integer $(0 \leq P X \leq 15)$, and then $-2+G X \leq$ $p_{x} \leq 2+G X$. Here, $G X$ represents the gravity point of object area in the input 2-D shading image. Decoding of $p_{y}$ is the same.

\section{Calculation of the Fitness Value of String}

Each string represents a modeled 3-D shape. The fitness value of the string is defined according to the similarity between the shading image determined by the 3 -D shape and the given (input) shading image as shown in figure 5 . The error between the two shading images is defined as

$$
\text { error }=\sum_{x} \sum_{y}\left|S_{i}(x, y)-S_{c}(x, y)\right|
$$

where $S_{i}$ is the input shading image and $S_{c}$ is the shading image calculated from the $3-\mathrm{D}$ shape represented by each string. The fitness value fitness is expressed as the following equation:

$$
\text { fitness }=\text { error }^{-1} \text {. }
$$

\section{Evolution}

The string having the maximum fitness value is sought by the evolutionary process which is described as follows.

The initial population of $n$ strings is first made by the random value. The population is reproduced for evolving into a population consist of strings with larger fitness $f$. While the reproduction of population, which is called as generation, is repeated, fitness values of strings in the population are increased. 


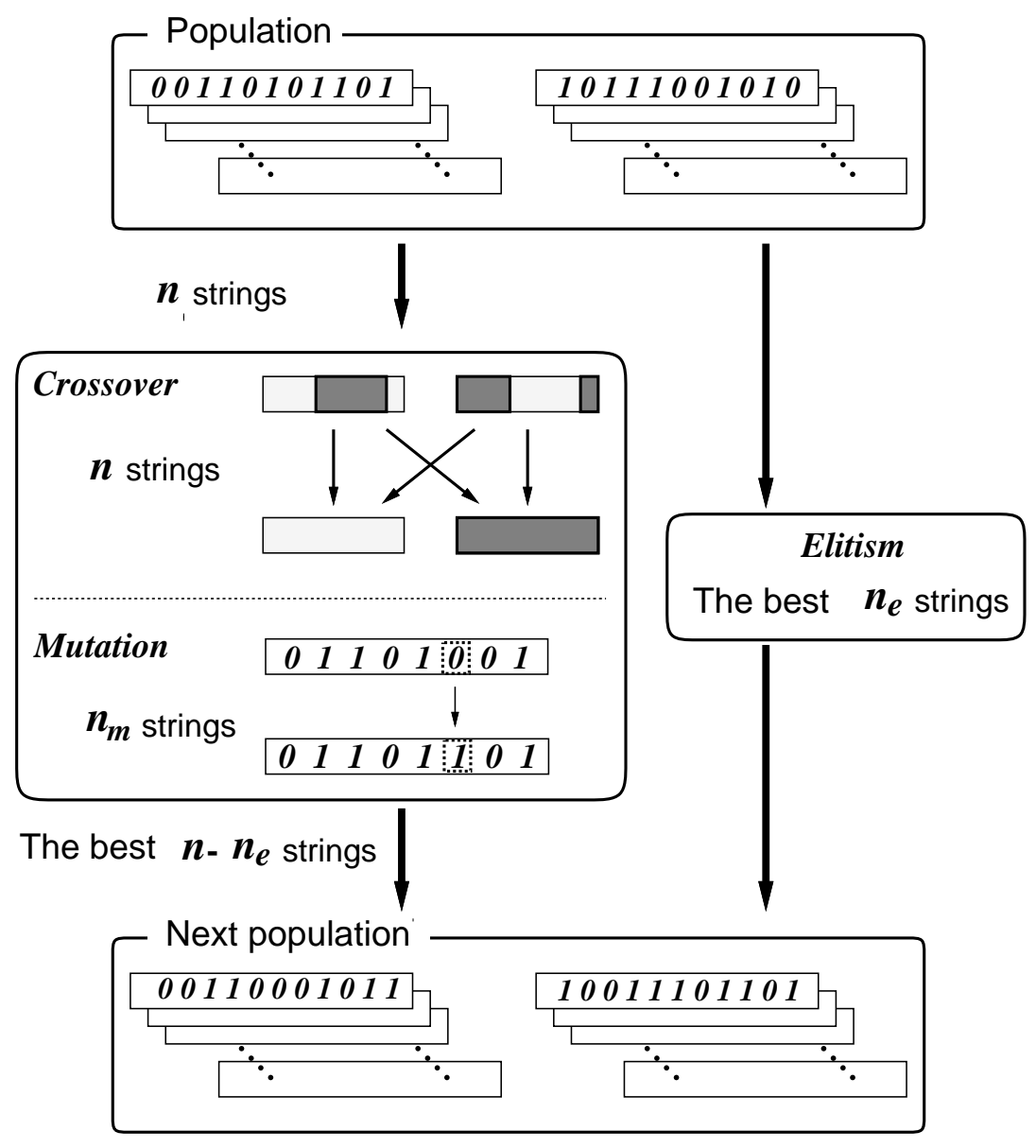

Figure 7: The flow of the evolutionary process in the proposed method.

Before the reproduction process, fitness values of strings in the population are calculated as show in figure 5 . The best $n_{e}$ strings are first copied into the next generation. This process is called as the elitist strategy [8]. Using the elitist strategy, evolution speed can be made faster. However, if the number of the elite strings $n_{e}$ is too large, the population tends to be trapped into a local minimum solution.

The rest strings of $\left(n-n_{e}\right)$ in the next population are reproduced by crossover operation. The pair of strings are selected according to the probability proportional to the fitness value. The two parents make two children by two-point crossover operation. The two point crossover can be regarded as exchanging a part of the ring of each string as shown in figure 6 .

After $n$ strings are reproduced by the crossover, $n_{m}$ strings are selected randomly from the $n$ strings for mutation. The mutation is performed by calculating exclusive OR between the string and a binary mask of the same length as the string which is randomly produced. The probability of bit "1" in the mask is determined by the mutation rate. The mutation is operated so that the population is not trapped into a local minimum.

From the $n$ strings reproduced by the crossover and the mutation, the best $\left(n-n_{e}\right)$ strings are remained into the next generation with the $n_{e}$ elite strings previously selected.

The flow of the process described above is shown in figure 7 . After repeating the processes, the parameters having the highest fitness value is considered as the estimated parameters representing the superquadrics model of 3-D shape.

\section{Computer Simulations}

To demonstrate the proposed method, superquadrics parameters were estimated from shading images obtained by computer (a) Input shading image.

(b) Output shading image.

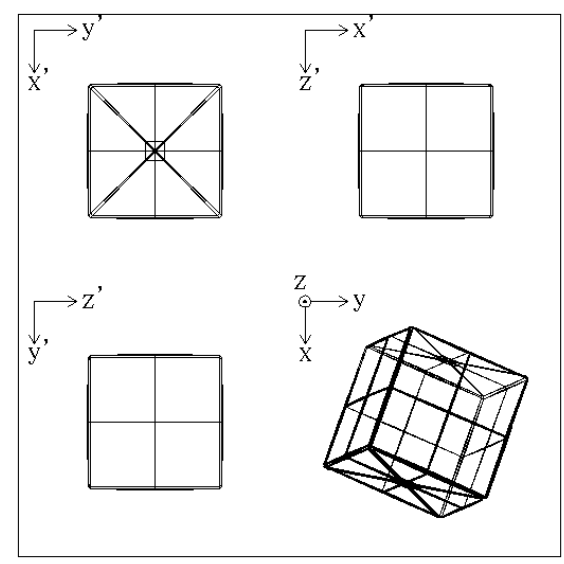

(c) Original shape.

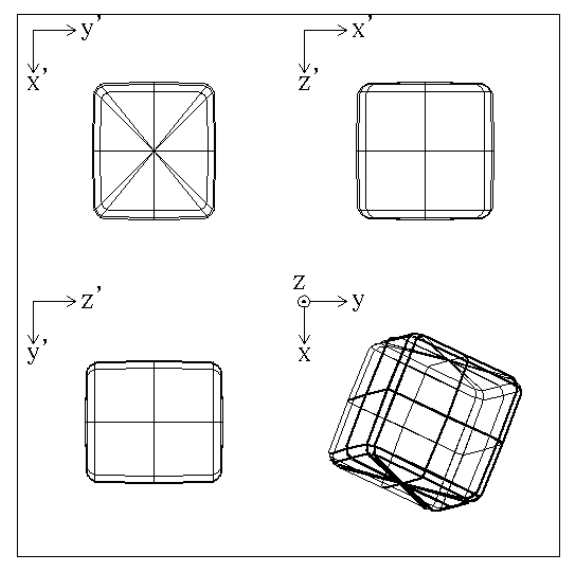

(d) Estimated shape.

\begin{tabular}{|c|c|c|c|c|c|c|c|}
\hline parameter & $a_{1}$ & $a_{2}$ & $a_{3}$ & $\varepsilon_{1}$ & $\varepsilon_{2}$ \\
\hline \hline original & 8.0 & 8.0 & 8.0 & 0.05 & 0.05 \\
\hline estimated & 8.13 & 7.34 & 8.13 & 0.19 & 0.19 \\
\cline { 3 - 7 } \\
\cline { 2 - 7 } & $\theta$ & $\phi$ & $\psi$ & $p_{x}$ & $p_{y}$ \\
\hline \hline $0.25 \pi$ & $0.38 \pi$ & $0.13 \pi$ & 0.0 & 0.0 \\
\hline & $0.57 \pi$ & $0.09 \pi$ & $0.86 \pi$ & 0.5 & -0.5 \\
\hline
\end{tabular}

(e) Comparison of estimated parameters with original parameters.

Figure 8: Results of parameter estimation in the case for cube.

simulations. The input 2 -D shading images was $32 \times 32$ pixels with 256 gray levels. The strings representing the model parameters were 64 bit binary. The number of the strings in a population $n$ was 500 . The number of the strings selected for the mutation in each generation $n_{m}$ was 50 . This means that the total mutation probability for each bit is $1 / 640\left(=n_{m} / n / 64\right)$. The generation was repeated 100 times. The number of the elite strings $n_{e}$ is 125 , which was experimentally determined.

Figures 8-11 show the input shading images (a), the output shading images calculated from the modeled shapes by the estimated parameters (b), 3-D shapes of the original superquadrics parameters (c), 3-D shapes of the estimated parameters (d), and the comparison of the estimated parameters with the original parameters (e).

In figure 13, the transition of error of the population in case for cube (figure 8) is shown. This indicates that the error is decreased effectively.

In accordance with these results, the estimated parameters are roughly close to the original. However, the estimation parameters of cube is not close to the original, although the reconstructed shape is close to the original as shown in figure 8 . This is caused by the fact that cubes can be represented by some parameters 
(a) Input shading image.

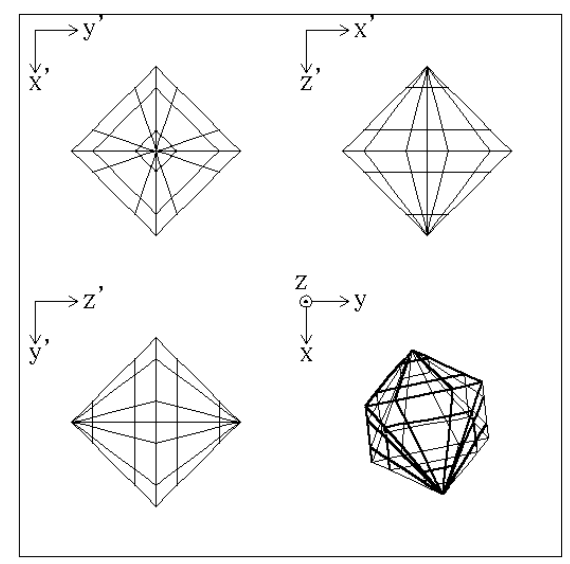

(c) Original shape. (b) Output shading image.

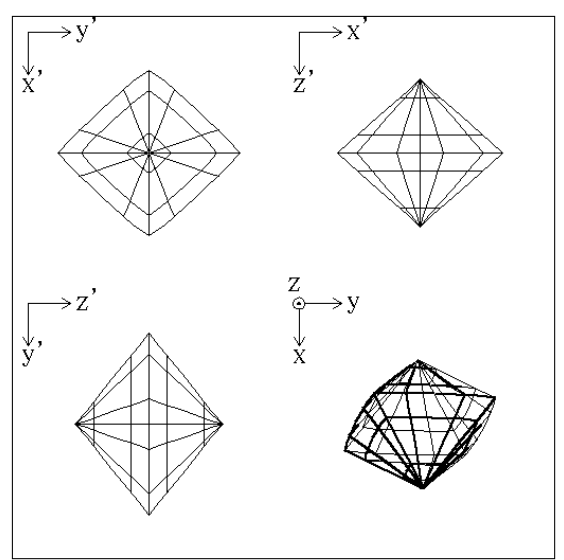

(d) Estimated shape.

\begin{tabular}{|c|c|c|c|c|c|c|c|c|}
\hline parameter & $a_{1}$ & $a_{2}$ & $a_{3}$ & $\varepsilon_{1}$ & $\varepsilon_{2}$ & & & \\
\hline original & 10.0 & 10.0 & 10.0 & 2.0 & 2.0 & & & \\
\hline estimated & 9.84 & 10.8 & 8.75 & 2.0 & 1.8 & & & \\
\hline & & & & $\theta$ & $\phi$ & $\psi$ & $p_{x}$ & $p_{y}$ \\
\hline & & & & $0.30 \pi$ & $0.20 \pi$ & $0.20 \pi$ & 0.0 & 0.0 \\
\hline & & & & $0.25 \pi$ & $1.73 \pi$ & $1.81 \pi$ & 0.0 & -0.5 \\
\hline
\end{tabular}

(e) Comparison of estimated parameters with original parameters.

Figure 9: Results of parameter estimation in the case for regular octahedron.

sets. As shown in figure 12 , cube shape can be expressed by both $\varepsilon=0.05$ and $\varepsilon=2.00$.

Although the estimation in eular angle parameters for each shape is different from the original, the estimation is considered as correct. The estimated parameters represent the same shape as the original, since there are some way in eular angle representing the same shape because of the axial symmetry of superquadrics.

\section{Conclusion}

In this paper, a new method for parametric reconstruction of 3D shape from 2-D shading image using genetic algorithm (GA) is proposed. In this method, superquadrics parameters of superquadrics 3-D model is estimated from 2-D shading image. For the estimation, the genetic algorithm is applied to an optimizing problem such that parameters providing the most conforming shading image to the input shading image are sought.

For demonstrating the performance of the proposed method, parameters of superquadrics model were estimated from shading images obtained by computer simulations. The results shows that the proper estimation was obtained because GA is robust for searching the best parameters.

(a) Input shading image.

(b) Output shading image.

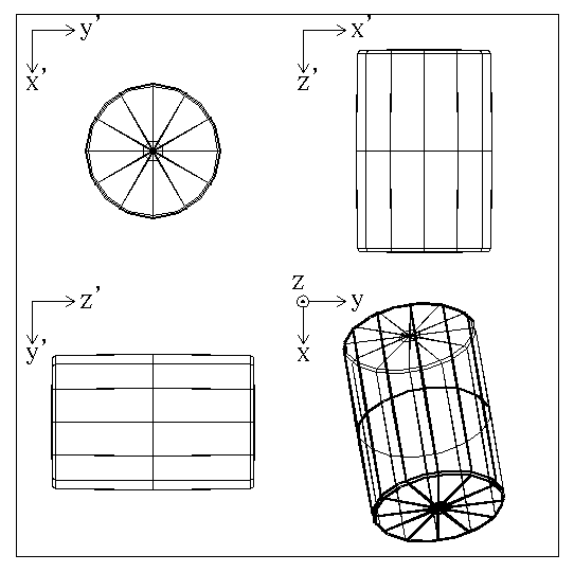

(c) Original shape.

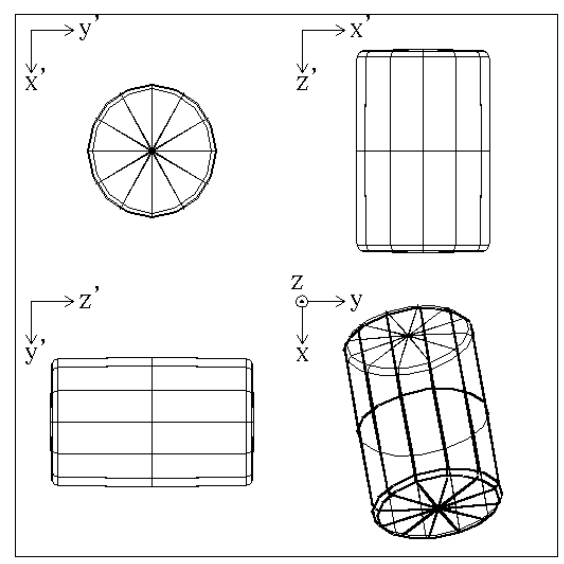

(d) Estimated shape.

\begin{tabular}{|c|c|c|c|c|c|c|c|c|}
\hline parameter & $a_{1}$ & $a_{2}$ & $a_{3}$ & $\varepsilon_{1}$ & $\varepsilon_{2}$ & & & \\
\hline original & 8.0 & 8.0 & 12.0 & 0.05 & 1.0 & & & \\
\hline estimated & 7.97 & 7.66 & 12.0 & 0.11 & 1.0 & & & \\
\hline & & & & $\theta$ & $\phi$ & $\psi$ & $p_{x}$ & $p_{y}$ \\
\hline & & & & $0.25 \pi$ & $0.25 \pi$ & $0.25 \pi$ & 0.0 & 0.0 \\
\hline & & & & $0.67 \pi$ & $1.08 \pi$ & $0.10 \pi$ & 0.0 & -0.5 \\
\hline
\end{tabular}

(e) Comparison of estimated parameters with original parameters.

Figure 10: Results of parameter estimation in the case for cylinder.

In this paper, we use the shading images that include only one object. In case for actual application, there exists some objects and some complicated objects in shading images. The application to such images is the next objective in future.

\section{ACKNOWLEDGMENTS}

The authors would like to express our appreciation to Prof. Shinji Ozawa and Prof. Masato Nakajima, Keio University, for valuable discussions.

\section{REFERENCES}

[1] B. K. P. Horn and M. J. Brooks, Shape from Shading, Cambridge, The MIT Press, 1989.

[2] H. Saito and K. Usami, "Shape from shading using genetic algorithm", Proc. of IECON'93, pp.1620-1625, 1993.

[3] A. P. Pentland, "Perceptual organization and the representation of natural form", Artifical Intelligence, pp.293-331, 1986. 
(a) Input shading image.

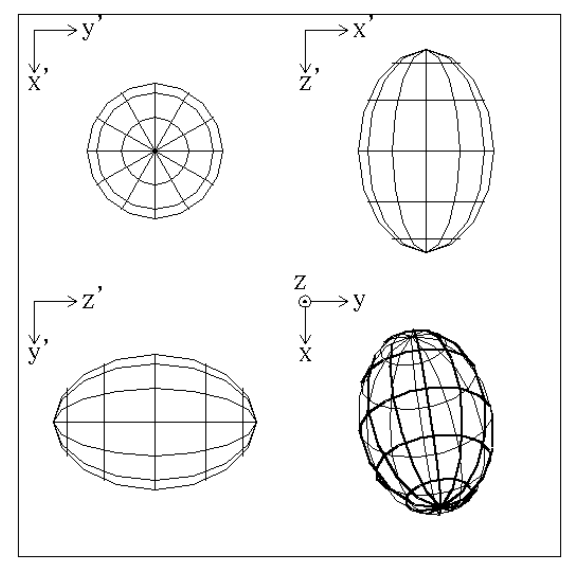

(c) Original shape. (b) Output shading image.

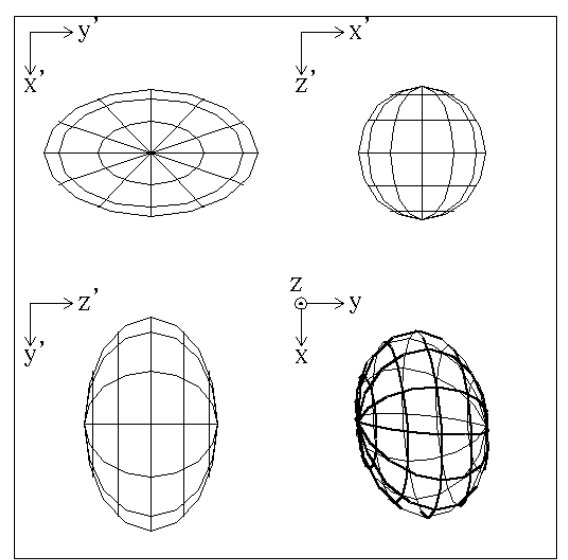

(d) Estimated shape.

\begin{tabular}{|c|c|c|c|c|c|c|c|}
\hline parameter & $a_{1}$ & $a_{2}$ & $a_{3}$ & $\varepsilon_{1}$ & $\varepsilon_{2}$ \\
\hline \hline original & 8.0 & 8.0 & 12.0 & 1.0 & 1.0 \\
\hline estimated & 7.50 & 12.7 & 7.97 & 1.0 & 1.0 \\
\hline \multicolumn{1}{|c|}{$\theta$} & $\phi$ & $\psi$ & $p_{x}$ & $p_{y}$ \\
\hline \hline $0.25 \pi$ & $0.25 \pi$ & $0.25 \pi$ & 0.0 & 0.0 \\
\cline { 2 - 7 } & \\
\cline { 2 - 7 } & $1.36 \pi$ & $1.71 \pi$ & $1.38 \pi$ & 0.0 & -0.5 \\
\hline
\end{tabular}

(e) Comparison of estimated parameters with original parameters.

Figure 11: Results of parameter estimation in the case for spheroid.

[4] A.H.Barr, "Superquadrics and angle-preserving transformations ", IEEE Comput. Graphics Applicat., vol.1, pp.11-23, 1981.

[5] A.H.Barr, "Global and local deformations of solid primitives ", Comput. Graphics, vol.18, No.3, pp.21-30, 1984.

[6] F. Solina and R. Bajcsy, "Recovery of parameteric models from range images : the case for superquadrics with global deformations", IEEE Trans. Patt. Anal. Machine Intell., vol. PAMI-12, No.2, pp.131-147, 1990.

[7] D. E. Goldberg, Genetic Algorithms in Search, Optimization, and Machine Learning, Addison-Wesley Publishing Company, 1989.

[8] L. Davis, Handbook of Genetic Algorithms, New York, Van Nostrand Reinhold, 1991.

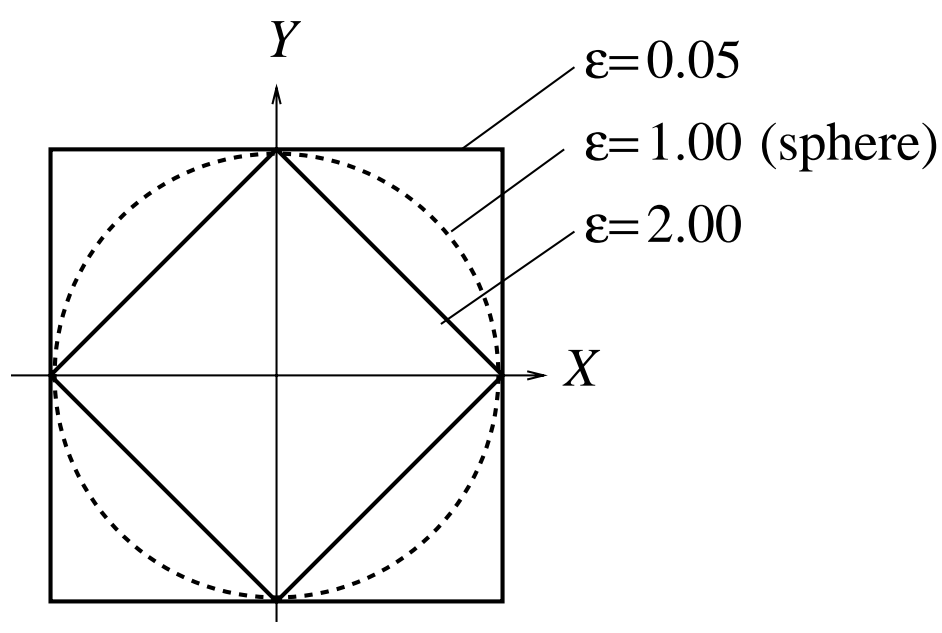

Figure 12: Schematic figure to explain that cubes can be represented by two sets of parameters. In this figure, both parameters $\varepsilon=0.05$ and $\varepsilon=2.00$ can represent cubes, although the orientations of two cubes are different from one another.

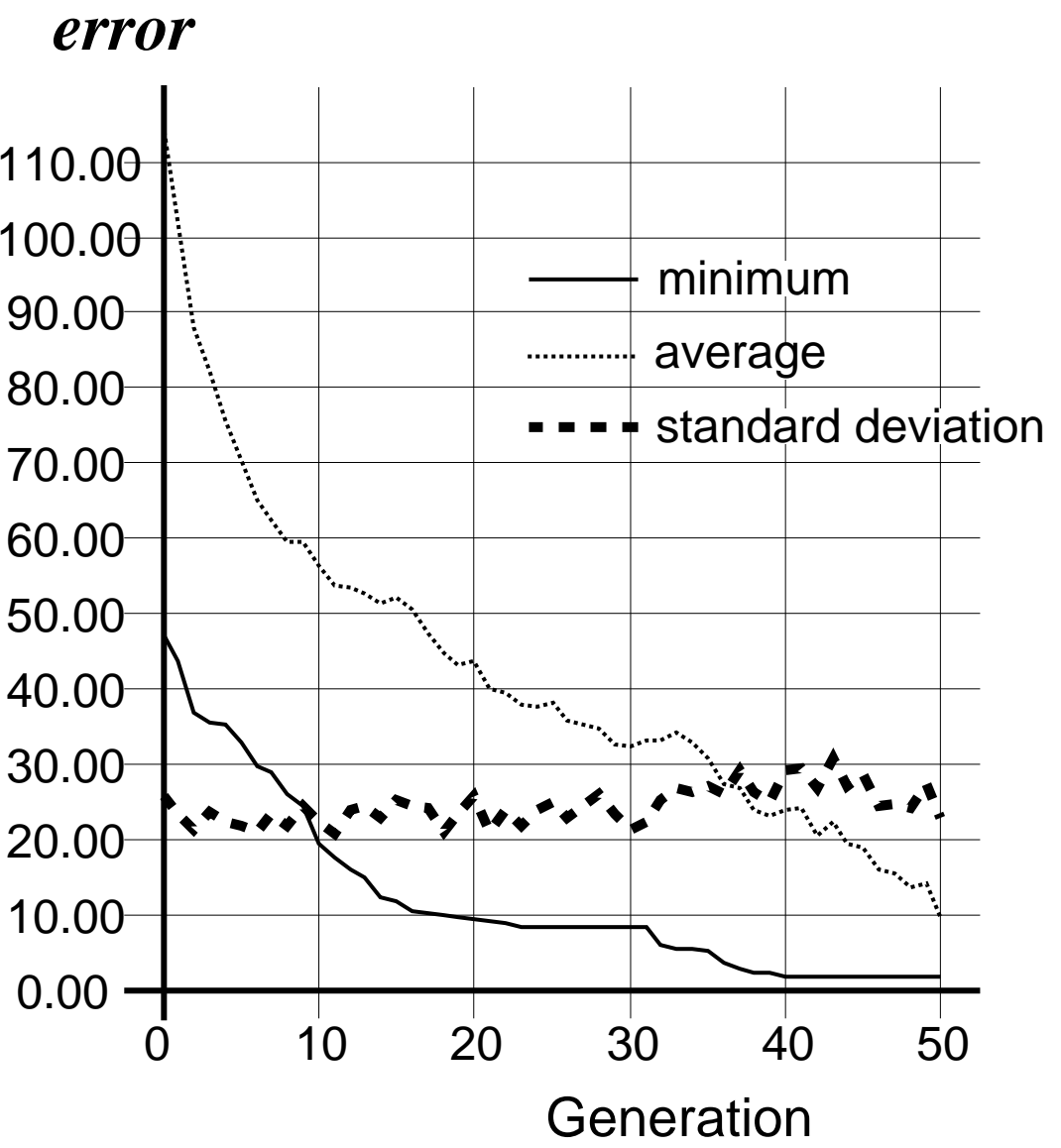

Figure 13: Transition of error in population for the case of estimating the cube parameters. 